\title{
Electrical-conduction mechanisms in polymer-copper-particle composites. I. Temperature and high-magnetic-field dependence of the conductivity
}

\author{
C. Pierre and R. Deltour \\ Physique des Solides, CP233, Université Libre de Bruxelles, boulevard du Triomphe, B-1050 Bruxelles, Belgium \\ J. A. A. J. Perenboom and P. J. M. Van Bentum \\ High-Field Magnet Laboratory and Research Institute for Materials, University of Nijmegen, Toernooiveld, \\ NL-6525 ED Nijmegen, The Netherlands
}

(Received 5 January 1990)

\begin{abstract}
A new method for the preparation of conducting copper-polymer composites is proposed. The origin of the electrical-conduction mechanism in these plastics is determined from an analysis of the temperature $(T=4-300 \mathrm{~K})$ and magnetic-field dependence $(B=0-15 \mathrm{~T})$ of the resistance. The observed saturation of the conductivity at high concentrations of the metallic particles is due to electrical transport across microcontacts between the copper particles. At low temperature these microcontacts are in the Sharvin regime (ballistic regime). This model for the electrical conduction can be extrapolated to similar systems and suggests ways to efficiently produce highly conductive plastic-metal composites.
\end{abstract}

\section{INTRODUCTION}

Highly conductive plastics have found numerous important industrial applications, and are used for heating devices, as antistatic materials, and for electromagnetic shielding. Among the properties that contributed to their success are their relatively low weight, ease of manufacture, and low cost. Despite their importance and great potential, relatively few studies of their fundamental properties have been carried out.

In this work we will report on our study of the electrical conductivity of conductive plastics with the aim of elucidating the conduction mechanisms in these systems. These studies have been carried out on extrinsically conductive plastics, which can be realized by dispersing conducting particles (metal particles, carbon black, etc.) in an insulating polymer. ${ }^{1,2}$ The particles are then incorporated in the polymer matrix by dispersion in the molten polymer and by cold or hot compression of the polymer-particle mixture, or by dispersion of the conducting particles in a thermohardening resin.

An alternative class of conductive plastics contains intrinisically conductive polymers and can be realized by chemically doping the polymer chains with either donors or acceptors. Examples include polyacetylene, polypyrolle, and polythiophene. ${ }^{3}$ Disordered conducting systems such as the polymer-conducting-particle mixtures studied in this work show, in general, an insulatorconductor transition at low particle concentrations, and the conduction pattern is very inhomogeneous and filamentary. This transition is well known as a percolation transition. At high particle concentrations the electrical conduction becomes homogeneous throughout the sample. The threshold values of particle volume concentration at which three-dimensional extrinsically conducting polymer systems become conducting can be as small as
$1-15 \%$, where the classical effective-medium theory will predict larger values. ${ }^{4}$ The study of this transition in realistic physical systems can provide a good test for recent theoretical predictions or computer simulations aimed at characterizing the percolation transition in disordered systems. In polymer-carbon-black composites one can observe the onset of electrical conduction well before the particles make physical contact, and this is attributed to tunneling, and different tunneling mechanisms have been proposed: charge energy-barrier hopping, simple tunneling of the charge carriers across the weak polymer barriers, and thermally activated hopping. ${ }^{5-9}$ More recently, the percolation transition was also studied through the presence of electrical flicker noise, and study of this noise in the percolation regime is a very selective way to put recent theories to the test. ${ }^{10}$

In the high-concentration regime the resistivity of the samples is observed to saturate at a value much greater than the resistivity of the pure conducting phase.

It is therefore very important to know which transport mechanism determines the conductivity and sets a limit on the conductivity in such conductive plastics. We have investigated the electrical conduction of highly concentrated particle systems by the measurements of the resistivity and its dependence on temperature and high magnetic fields. In the polymer-copper-particle mixtures we have found that the conductivity is principally determined by the properties of the microscopic contacts between neighboring particles.

\section{ELECTRICAL CONDUCTION IN COMPOSITE MATERIALS}

Electrical conduction in composite materials has been studied for a very long time: Already at the end of last century Maxwell ${ }^{11}$ was interested in the conductive properties of an homogeneously conducting system embedded 
with highly resistive spherical particles.

The theoretical study of conduction in composite materials has more recently focused on numerically soluble models $^{12-14}$ and computer simulations. ${ }^{15-19}$

On the experimental front, Gurland ${ }^{20}$ led the first pioneering studies of the electrical behavior of composites made by randomly dispersing metallic (silver) particles in Bakelite powder. He observed an insulatorconductor transition at a particle volume concentration of $37 \%$. The very steep variation of the conductivity is correlated to the average number of electrical contacts, $b$, which becomes critical at the percolation threshold ( $b_{c}=1.3-1.5$ for the three-dimensional case).

In bond-percolation analysis, this number is now known as an approximate invariant only affected by the dimensionality $d$ of the network: $b_{c} \approx d /(d-1)=z p_{c}$, where $z$ is the coordination number of the resistance network considered and $p_{c}$ the critical bond-percolation probability.

Malliaris and Turner ${ }^{21}$ and later Bhattacharya ${ }^{22}$ observed much lower threshold values, of the order of $5-6 \%$, in systems obtained by the compaction of polyethylene powders with nickel or polyvinylchloride with copper.

These composite materials are very different from the above-mentioned system and are characterized by the segregation of the metallic particles (of radius $R_{m}$ ) at the interface between the polymer particles (of radius $R_{p}$ ). Thus, segregation is responsible for the low percolation threshold. The critical volume diminishes with the increase of the ratio $R_{p} / R_{m}$. In Gurland's experiments the two types of particles (metallic and polymer) have the same shape and the same size, i.e., a copper particle has the same probability of occupying a position of the space as a polymer particle.

The critical volume $V_{c}$ can be estimated, in terms of the critical site-percolation probability $X_{c}$ and the filling factor $f$ of a resistance network, by $V_{c}=f X_{c}$.

In the work of Malliaris and Turner, ${ }^{21}$ this probability is increased by the restriction on the number of space points available for the metallic phase. De Araujo and Rosenberg have performed experiments ${ }^{23}$ with epoxy resin randomly loaded with $\mathrm{Cu}, \mathrm{Ag}, \mathrm{Al}, \mathrm{Sn}$, or $\mathrm{Pb}$ particles, and have observed conductivity threshold values close to $40 \%$, while Aharoni, ${ }^{24}$ experimenting on similar systems, found values around $20 \%$.

Such differences can easily be explained by the granulometric distribution. At the same total volume of conducting particles, a system with a broad granulometric distribution has a lower probability of realizing intergranular electrical contacts than a system with a more homogeneous particle distribution. The threshold value for $V_{c}$ is also very sensitive to the random character, the shape of the particles, and the conduction mechanism relevant for the system considered.

Important information on the conductor-insulator transition comes from the variation of the conductivity $\sigma$ :

$$
\sigma(V)=\sigma_{0}\left(V-V_{c}\right)^{t}, \quad V \geq V_{c}
$$

where $t$ is the critical exponent characterizing the vanishing of the conductivity at the percolation threshold. The invariant properties of the $t$ exponent are much more restrictive than the critical volume for the different systems studied.

In the discrete-percolation case, when one only considers the geometric problem of establishing continuous conducting paths through the sample, this exponent depends essentially on the dimensionality of the system of particles. In such a problem the particles are either in contact or not.

In real systems, as in the polymer-conducting-particle composites, some of the particles can be oxidized, for example, and thus give rise to a distribution of values of the contact conductance. The problem should then be analyzed as a continuous percolation. Recently, Balberg ${ }^{25}$ has shown in polymer-carbon-black systems that the particles' morphology influences the distribution of distances separating particle aggregates. Spherical particles give a broad distribution of intergranular separations. As tunneling conduction is very sensitive to the distances between aggregates, values of the critical exponent $t$ can be as high as $t \approx 4$ (and one approaches the continuous percolation problem). For the very irregularly shaped carbon-black particles, the distribution of intergranular distances is narrower and one gets value of the exponent $t$ between 1.6 and 1.7, close to the discrete-percolation value in three dimensions. The continuous-percolation model can be applied to other systems such as the fluidflow experiments in a porous medium. ${ }^{26,27}$

\section{SAMPLE CHARACTERIZATION AND MEASUREMENT TECHNIQUE}

The conductive plastic is produced by dispersing cleaned copper particles in polystyrene. Copper will rapidly oxidize even at room temperature, ${ }^{28}$ so the copper powders obtained from commercial sources cannot be used directly.

Several different oxide-cleaning procedures have been tried out to eliminate the oxide coating on the copper particles, and their effectiveness has been compared with respect to the conductivity values obtained after their incorporation into the polystyrene: reduction with hydrogen, and chemical attack with nitric acid or hydrochloric acid solution. This last method was selected as the most effective.

Hydrogen reduction was at a disadvantage, because it leads to sintering of the powder. Cleaning in nitric acid solution is not satisfactory because of the competition between the acid and the oxidizing character of the reagent.

We have used two copper powders of different shape (spherical, irregular), but with the same granulometric distribution (see inset of Fig. 1, average diameter 12-15 $\mu \mathrm{m})$. This permitted us to compare the shape effect on the conductivity values of the composite.

The cleaned copper powder is brought into a xylenepolystyrene solution without exposure to the air and at room temperature. This solution has strong deflocculating properties and allows an homogeneous dispersion of the powder in the polymer matrix. ${ }^{29}$ The 


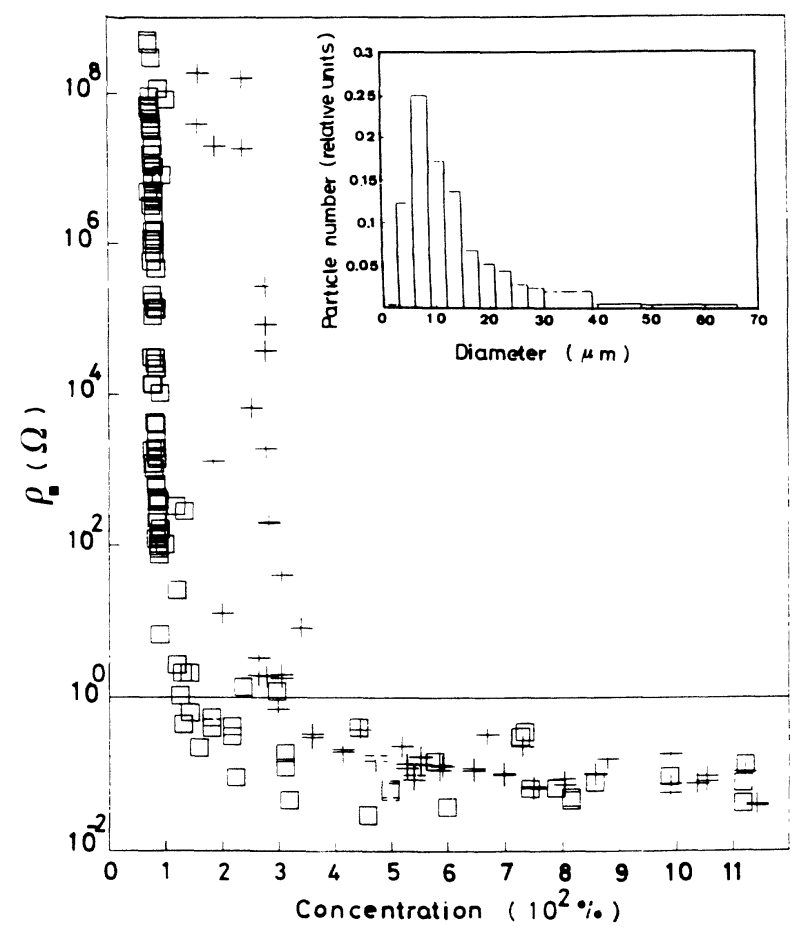

FIG. 1. Percolation transition in the dependence of the resistivity per unit area of the copper-particle-polymer composites on the particle surface concentration. $\square$, irregular powder; + , spherical powder. The inset shows the particlediameter-distribution histogram of the spherical powder.

solution is deposited on $5 \times 5-\mathrm{cm}^{2}$ glass slides. After slow evaporation of the solvent in ambient atmosphere over a few days and in a vacuum of $10^{-3}$ Torr for $1 \mathrm{~d}$ to remove any solvent traces that could give rise to ionic currents, ${ }^{30}$ we obtained films with a two-dimensional particle distribution [see Fig. 2(a)], slightly transparent at the percolation threshold. The samples are conductive on one surface of the film only. In the high-concentration limit the particles fill the film over its entire thickness and the conduction is three dimensional [Fig. 2(b)].

Measurements of the electrical resistance were made with the usual four-probe technique. Electrical contacts were made by copper electrolysis, which produced a reliable and intimate contact with the conducting path in the polymer composite. For temperature-dependent measurements we used a helium-flow cryostat. For the transverse magnetoresistance measurements, Bitter magnets (maximum field in this study of $15 \mathrm{~T}$ in $6-\mathrm{cm}$ bore) were used. For those measurements we inserted the samples into a bath cryostat cooled with nitrogen or liquid helium. In this manner we could avoid temperature drifts that could possibly have given resistance variations in excess of the small magnetoresistance variation we wanted to measure.

Two different measuring techniques allowed us to measure $0.01 \%$ variations in resistance. The first method was the usual synchronous detection used to measure weak nonlinearities in resistance. With the second method, the $\mathrm{dc}$ voltage across the sample was measured with a Keith-
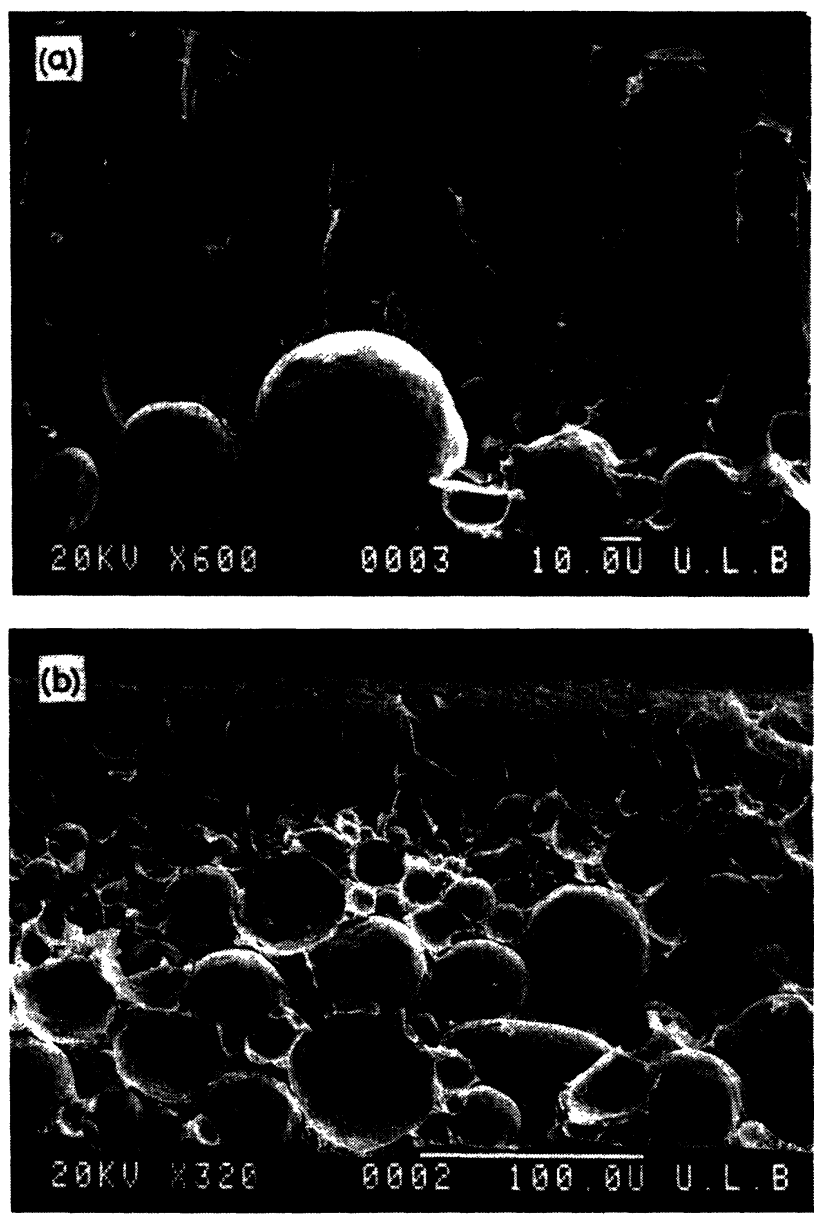

FIG. 2. Cross-sectional views of copper-particle-polymer samples: (a) low-particle-concentration limit (percolationthreshold limit); (b) moderately high particle concentration, just before the particles fill the entire film thickness.

ley model 181 nanovoltmeter connected with twisted and uninterrupted copper wires to the electrodes on the sample. The current direction through the sample was inverted three or four times, and the values $V^{+}$and $V^{-}$, extrapolated on a $X Y$ recorder to the inversion times, allowed us to eliminate temperature-dependent thermoelectrical effects important in the measurements on the compact powders (low-resistance values, $R=10^{-5} \Omega$ ).

\section{RESULTS}

Figure 1 shows the conductor-insulator percolation transition in units of resistivity per unit area, as the surface particle concentration is reduced. Data for two powders of different grain shapes are presented: + for spherical particles and $\square$ for nonspherical particles.

The surface concentrations at the percolation threshold, $S_{c \text {,spherical }}=147 \%$ (i.e., $1 \frac{1}{2}$ layers) and $S_{c \text {,irregular }}$ $=77 \%$, are higher than the values expected for discrete percolation, i.e., $S_{c} \approx 50 \%$. The percolation curve is not only determined by the number of conducting paths that will diminish with lowering of the particle concentration, 
but also by the number of particle layers over the width of the polymer film. This made it impossible to experimentally determine the critical transition exponent $t$ [Eq. (1)] by simply varying $S$.

It is also noteworthy that the value of the conductivity obtained in the high-concentration limit is between 100 and 1000 times higher than the values found in the literature for similar systems $\left[\sigma \approx 10^{3}-10^{2}(\Omega \mathrm{m})^{-1}\right],{ }^{1,20-24}$ without applying any pressure. This improvement is due to the low-temperature solvent-preparation method, to the weak affinity of the polymer for copper, to the copper-particle cleaning method, and to the presence of oxidation inhibitors in the industrial polymer used. ${ }^{31}$

The limiting resistivity of the highly concentrated composite films, however, is found to be $10^{3}-10^{4}$ times higher than for pure metallic copper $\left(\rho=1.7 \times 10^{-8} \Omega \mathrm{m}\right)$. The observed high values for the saturation of $\rho$ and for the percolation threshold suggest a poor conduction mechanism dominated by contact between particles. These contacts are small compared to the radius $r$ of the particles and their size can be estimated from the Hertz formula ${ }^{32}$ for the radius of contact, $a$, between spherical particles:

$$
a=(\operatorname{Pr} / E)^{1 / 3} \approx 1.3 \times 10^{-9} \mathrm{~m},
$$

where $P$ is the load applied to the contact (proportional to the weight of a copper particle) and $E$ is the Young modulus of copper. The value of $a$ in Eq. (2) corresponds to a typical contact size calculated with $r=6 \mu \mathrm{m}$.

Observation with optical and electronic scanning microscopy shows a random close packing of the particles (see, for example, Fig. 2). The volume occupied by the particles is then close to $52 \%$, the value expected for a close-packed cubic lattice. ${ }^{33}$ The total resistance $R$ of such a lattice as a function of the contact resistance $\left(r_{\text {contact }}\right)$ is given by

$$
R=r_{\text {contact }} / L^{d-2},
$$

where $L$ is the length of the system normalized to the particle radius and $d$ the dimensionality of the lattice.

In our highly concentrated samples, different mechanisms for electrical conduction between the particles may be present: metallic contacts, tunneling (as is observed in other similar systems at the percolation transition), and conduction through oxidized contacts.

Table I gives an estimate of the average contact resistance $r_{\text {contact }}$ using Eq. (3) with $d=3$ and the value of resistance $R$ for the composite samples of spherical and irregular copper particle powders. The values in Table I are typical for the resistance of a point contact in the ballistic regime, when the mean free path of the electrons, $l$, is greater than the radius of the contact $(l>>a)$ (Ref.

TABLE I. Estimate of interparticle contact radius.

\begin{tabular}{llccc}
\hline \hline Powder & $\begin{array}{c}r_{\text {contact }}=R_{S 0} \\
(\Omega)\end{array}$ & $\begin{array}{c}l_{\mathrm{Cu}}(4 \mathrm{~K}) \\
(\mathrm{nm})\end{array}$ & $\begin{array}{c}a_{S 1} \\
(\mathrm{~nm})\end{array}$ & $\begin{array}{c}a_{S 2} \\
(\mathrm{~nm})\end{array}$ \\
\hline spherical & 7.4 & 37 & 6.15 & $2.3-7.2$ \\
irregular & 1.75 & 210 & 13 & 11 \\
\hline \hline
\end{tabular}

34) and cannot be understood in terms of tunneling or conduction through oxidized contacts. The latter two mechanisms would produce higher resistance values. In Table I we have also given the low-temperature $(4 \mathrm{~K})$ values for $l$ estimated from the measured resistivity of the two copper powders, tightly compressed (at $p=5 \times 10^{8}$ $\mathrm{Pa})$ in the absence of the polymer. The difference between the mean free paths for the different powders, nearly a factor of 6 , is interpreted as due to dislocations introduced in the manufacturing process.

In the ballistic regime the contact resistance, $r_{\text {contact }}$, can be estimated from the Sharvin expression, $R_{S 0}$, and can be written as ${ }^{35-37}$

$$
r_{\text {contact }}=R_{S 0}=\frac{4}{3 \pi} \frac{\rho l}{a^{2}} .
$$

In the third column of Table I an estimate is given of the contact radius $a_{S 1}$ obtained using Eq. (4). According to Eq. (4), and because the resistivity $\rho$ is inversely proportional to the mean free path, the contact resistance must be independent of temperature $T$ and applied magnetic field $B$ :

$$
\frac{R_{S 0}(T, B)-R_{S 0}(T=4 \mathrm{~K}, B=0)}{R_{S 0}(T=4 \mathrm{~K}, B=0)} \approx 0 .
$$

Figures 3 and 4 show, for comparison, the variations of the resistivity with temperature and with magnetic field of a highly concentrated composite sample and of a sample of pure compressed powder. At low temperatures the

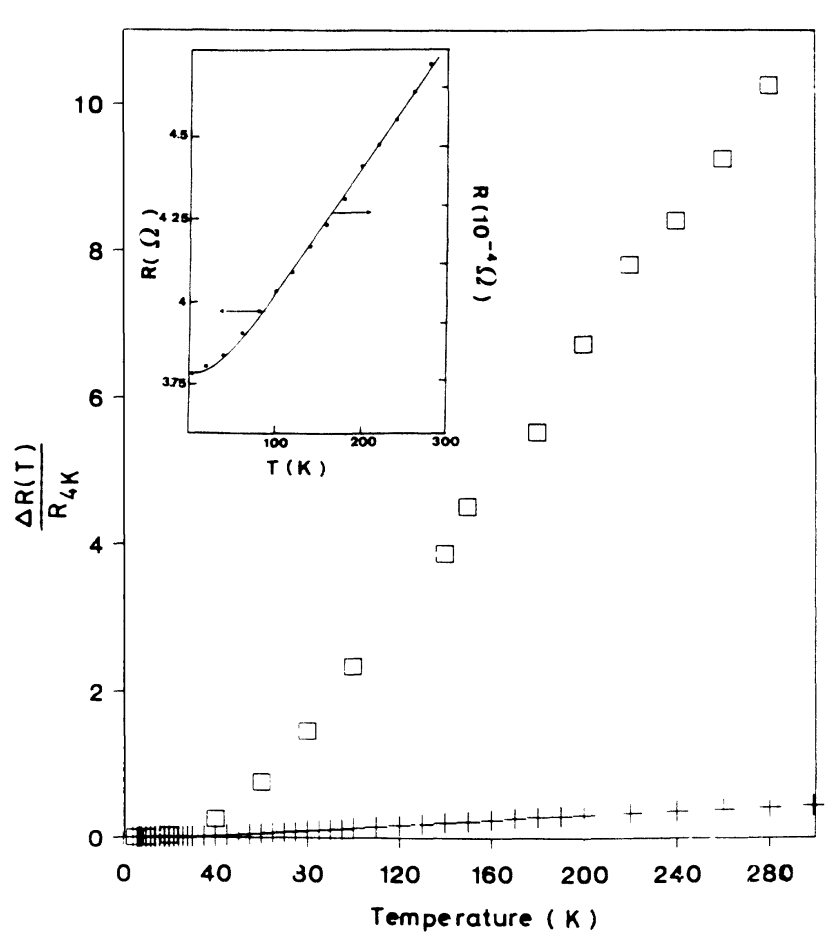

FIG. 3. Variation with temperature of the resistance of copper-particle-polymer samples: + , composite material; $\square$, compressed powder. The inset shows that apart from a scale factor the temperature dependence is the same for both samples ( $\bigcirc$, composite material; solid line, compressed powder). 


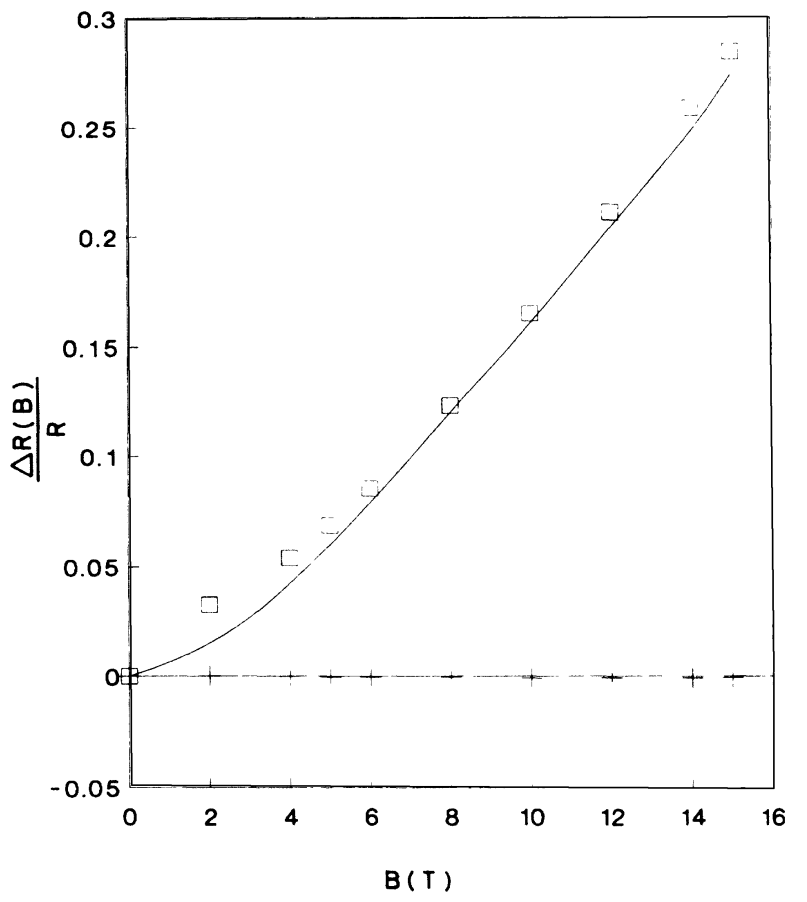

FIG. 4. Variation in magnetoresistance $\Delta R(B) / R(0)$ observed at $T=4.2 \mathrm{~K}$ in the pure compressed powder ( $\square$ ), compared to the very weak variation observed in a highly concentrated copper-particle-polymer composite sample $(+)$. The solid line represent results of Ref. 41 for the magnetoresistance of polycrystalline copper.

magnetic field dependence of the resistance of the composite is found to be negligible, in agreement with Eq. (5); however, significant variations of resistance with temperature are observed (between $25 \%$ and $45 \%$ ), depending on the type of powder and its concentration in the matrix.

From the inset of Fig. 3, which also shows the temperature variation of the resistance of pure copper (measured in the tightly compressed pure powders), it can be seen that the temperature dependence of the resistance of the composite material is quite similar and that, therefore, the same mechanism as in the bulk, i.e., the electronphonon interaction, must cause this temperature dependence. This characteristic temperature dependence of the resistivity of pure copper definitively eliminates tunneling as the possible principal conduction mechanism. The Sharvin contact resistance does show some temperature dependence not taken into account in Eq. (5). Indeed, including electron-phonon scattering, one finds ${ }^{35,37}$

$$
R_{S}(T)=R_{S 0}+\frac{32}{9 \pi^{2}} \frac{\rho(T)}{a} .
$$

From Eq. (6) one can get a second independent estimate of the average dimension of the contact $a_{S 2}$, and these values are also listed in Table $I$. The agreement with the other estimate of the contact dimension is remarkable when one considers the great number of such contacts in the system and the fact that this last estimate of $a$ is in- dependent of possible inhomogeneities of the conducting paths through the sample. Indeed, let us make the reasonable assumption that there is no variation of the number of conducting paths through the sample when the sample is cooled.

We find that the ratio of the contact dimension of the two powders $(\approx 2)$ is the inverse of the ratio of the critical concentrations $S_{c}$ at the percolation threshold for the different sample types (irregular or spherical particles). This shows that the percolation transition in these systems depends more on the type of contact and the mechanism of conduction (i.e., continuous percolation) than on the geometric interconnection probability at the threshold (i.e., discrete percolation). As can be seen in Fig. 4, we have observed a variation of the resistance of $\approx 28 \%$ in magnetic fields up to $15 \mathrm{~T}$ and at low temperature for compressed (spherical and irregular) powders. The observed linear field dependence at low temperature can be explained in the high-field limit for electron transport (when $\omega_{c} \tau \gg 1$, where $\omega_{c}$ is the cyclotron frequency and $\tau$ the electronic relaxation time). ${ }^{38-40}$

Also in Fig. 4 we have compared the measurements of Lüthi ${ }^{41}$ on pure polycrystalline copper with out data for the irregular compressed powder. The experimental error is smaller than the symbols in the graph. The good agreement between these data also support our interpretation of the results on the high-concentration irregular composite samples previously discussed.

On the other hand, for spherical-particle powders there is a slight difference with the variation of the resistivity of bulk copper. This may be related to the less satisfactory compaction of the spherical-particle powder, ${ }^{42}$ which can lead to an overestimate of the resistivity ratio used for the comparison with the results of Ref. 41 .

At high temperature, however, we find quadratic magnetic field dependence of the order of a few tenths of a percent, typical for pure copper in this temperature range. In this case the contact resistance will be limited by the electron mean free path and is given by Maxwell's expression $(l<<a)$ (Refs. 32, 37, and 43):

$$
R=\rho / 2 a,
$$

and the magnetic field dependence of the resistance will be, as in pure copper, $\rho_{\mathrm{Cu}}(B)$ :

$$
\frac{\Delta R(B)}{R}=\frac{\Delta \rho_{\mathrm{Cu}}(B)}{\rho_{\mathrm{Cu}}} .
$$

This model for the heavily loaded copperparticle-polystyrene composites can be extrapolated to other conducting-particle-polymer systems.

In the case of $2-\mu \mathrm{m}$ graphite sheets dispersed in polystyrene, Quivy et al. ${ }^{44}$ have carried out similar measurements at low temperature and in high magnetic fields. In their case they observed a supplementary term in the Sharvin contact resistance due to the two-dimensional character of conduction in graphite.

In some experiments we carried out on a moderately high concentration of carbon-black-polymer systems, we have also found temperature variations similar to pure carbon black. This is not surprising given the strong ten- 
dency for the particles to form long continuous aggregates, which makes these systems well conducting with a very low percolation threshold. ${ }^{2}$

\section{CONCLUSIONS}

We have developed a preparation method of reproducible, highly conductive copper-particle-polystyrene composites, and have shown on the basis of magnetic field and temperature dependence that the conductivity in the high-concentration limit can be explained by electron transport through Sharvin-type metallic microcontacts.

We suggest that four important points be considered to achieve high-conductivity values in polymer-metal composites.

(1) A good oxide-cleaning method for the metallic powders.

(2) The introduction of the powder in the polymer without temperature elevation and exposure to air. The high-pressure compression usually done in industrial manufacturing must be realized after the particles are incorporated into the polymer matrix.

(3) The choice of a polymer with weak affinity for the metal considered. The polymer solvent dispersion technique seems to be good because it promotes intimate metal-metal contact after the solvent evaporation to the detriment of the polymer-metal interface.

(4) Finally, the use of the most irregularly shaped particles that permit large stable electrical contacts linking the particles.

\section{ACKNOWLEDGMENTS}

We would like to thank Professor G. Geuskens, Professor R. Rammal, and P. Wyder for valuable discussions and suggestions. This work was supported by the Institut pour la Recherche Scientifique dans I'Industrie et l'Agriculture (for C.P.), the European Economic Communities under Contract No. ST280, and the Communauté Française de Belgique.
${ }^{1}$ Metal-Filled Polymers, edited by Swapan K. Bhattacharya (Dekker, New York, 1986).

${ }^{2}$ Carbon-Black Polymer Composites, edited by E. K. Sichel (Dekker, New York, 1982).

${ }^{3}$ Y.-W. Park, A. J. Heeger, M. A. Druy, and A. G. MacDiarmid, J. Chem. Phys. 73, 946 (1980).

${ }^{4}$ F. Bueche, J. Appl. Phys. 43, 4837 (1972); 44, 532 (1973); J. Polym. Sci. 11, 1319 (1973).

${ }^{5}$ B. Abeles, P. Sheng, M. D. Coutts, and Y. Arie, Adv. Phys. 24, 407 (1975).

${ }^{6} \mathrm{~J} .-$ B. Donnet and A. Voet, Carbon Black (Dekker, New York, 1976).

${ }^{7}$ E. K. Sichel and J. I. Gittleman, J. Electron. Mater. 11, 699 (1982).

${ }^{8}$ P. Sheng and J. Klafter, Phys. Rev. B 27, 2583 (1983).

${ }^{9}$ M. Mehbod, P. Wyder, R. Deltour, C. Pierre, and G. Geuskens, Phys. Rev. B 36, 7627 (1987).

${ }^{10}$ R. Rammal, C. Tannous, and A.-M. S. Tremblay, Phys. Rev. A 31, 2662 (1985).

${ }^{11}$ Electrical Transport and Optical Properties of Inhomogeneous Media (Ohio State University, 1977), Proceedings of the First Conference on the Electrical Transport and Optical Properties of Inhomogeneous Media, AIP Conf. Proc. No. 40, edited by J. C. Garland and D. B. Tanner (AIP, New York, 1978).

${ }^{12}$ Percolation Structures and Processes, edited by G. Deutscher, R. Zallen, and J. Adler [Ann. Israel Phys. Soc. 5 (1983)].

${ }^{13}$ A. G. Dum, J. W. Essam, and J. M. Loveluck, J. Phys. C 8, 4219 (1975).

${ }^{14}$ M. F. Sykes, D. S. Gaunt, and M. Glen, J. Phys. A 9, 87 (1976); 9, 97 (1976); 9, 715 (1976); 9, 727 (1976); 9, 1109 (1976); 9, 1705 (1976).

${ }^{15}$ S. Kirkpatrick, Phys. Rev. Lett. 36, 69 (1976).

${ }^{16}$ S. Kirkpatrick, Rev. Mod. Phys. 45, 574 (1973).

${ }^{17}$ Ill-Condensed Matter, edited by R. Balian, R. Maynard, and G. Toulouse, Les Houches Session XXXI (North-Holland, Amsterdam, 1978); also see Chance and Matter, edited by J. Souletie, J. Vannimenus, and R. Stora, Les Houches Session XLVI (North-Holland, Amsterdam, 1978).

${ }^{18}$ V. K. S. Shante and S. Kirkpatrick, Adv. Phys. 20, 325 (1971).

${ }^{19}$ G. E. Pike and C. H. Seager, Phys. Rev. B 10, 1421 (1974); 10, 1435 (1974).

${ }^{20}$ J. Gurland, Trans. Metall. Soc. AIME 236, 642 (1966).
${ }^{21}$ A. Malliaris and D. T. Turner, J. Appl. Phys. 42, 614 (1971).

22J. Swapan and K. Bhattacharya, Polymer 20, 116 (1979).

${ }^{23}$ F. F. T. de Araujo and H. M. Rosenberg, J. Phys. D 9, 1025 (1976).

${ }^{24}$ S. M. Aharoni, J. Appl. Phys. 43, 2463 (1972).

${ }^{25}$ I. Balberg, Phys. Rev. Lett. 59, 1305 (1987), and references therein.

${ }^{26}$ Po-Zen Wong, J. Koplik, and J. P. Tomanic, Phys. Rev. B 30, 6606 (1984).

${ }^{27}$ J. N. Roberts and L. M. Schwartz, Phys. Rev. B 31, 5990 (1985).

${ }^{28} \mathrm{U}$. R. Evans, The Corrosion and Oxidations of Metals: Scientific Principles and Practical Applications (Arnold, London, 1960).

${ }^{29}$ T. Allen, Particle Size Measurement (Powder Technology Series), 2nd ed. (Chapman and Hall, London, 1975).

${ }^{30}$ J. P. Reboul, J. Appl. Phys. 46, 2961 (1975).

${ }^{31}$ C. Pierre and R. Deltour, Patent No. BE 8900266.

${ }^{32}$ R. Holm, Electric Contacts Handbook, 3rd ed. (SpringerVerlag, Berlin, 1958).

${ }^{33}$ R. M. Scarisbrick, J. Phys. D 6, 2098 (1973).

${ }^{34}$ Yu V. Sharvin, Zh. Eksp. Teor. Fiz. 48, 984 (1965) [Sov. Phys. - JETP 21, 655 (1965)].

${ }^{35}$ A. G. M. Jansen, Ph.D. thesis, University of Nijmegen, Nijmegen, The Netherlands, 1980 (unpublished).

${ }^{36}$ A. I. Akimenko, A. B. Verkin, and I. K. Y. Yanson, J. Low Temp. Phys. 54, 247 (1984).

${ }^{37}$ G. Wexler, Proc. Phys. Soc. London 89, 927 (1966).

${ }^{38} \mathrm{~J}$. J. L. Olsen, in Electron Transport in Metals, Vol. 12 of Interscience Tracts on Physics and Astronomy, edited by (Interscience, New York, 1962).

${ }^{39}$ C. Kittel, Quantum Theory of Solids (Wiley, New York, 1963).

${ }^{40}$ G. J. C. L. Bruls, Ph.D. thesis, University of Nijmegen, Nijmegen, The Netherlands, 1985 (unpublished).

${ }^{41}$ B. Lüthi, Helv. Phys. Acta 33, 161 (1960).

${ }^{42}$ C. G. Goetzel, Treatise on Powder Metallurgy 1 (Interscience, New York, 1949).

${ }^{43}$ J. C. Maxwell, A Treatise on Electricity and Magnetism, 1891 (Dover, New York, 1954).

${ }^{44}$ A. Quivy, R. Deltour, A. G. M. Jansen, and P. Wyder, Phys. Rev. B 39, 1026 (1989). 

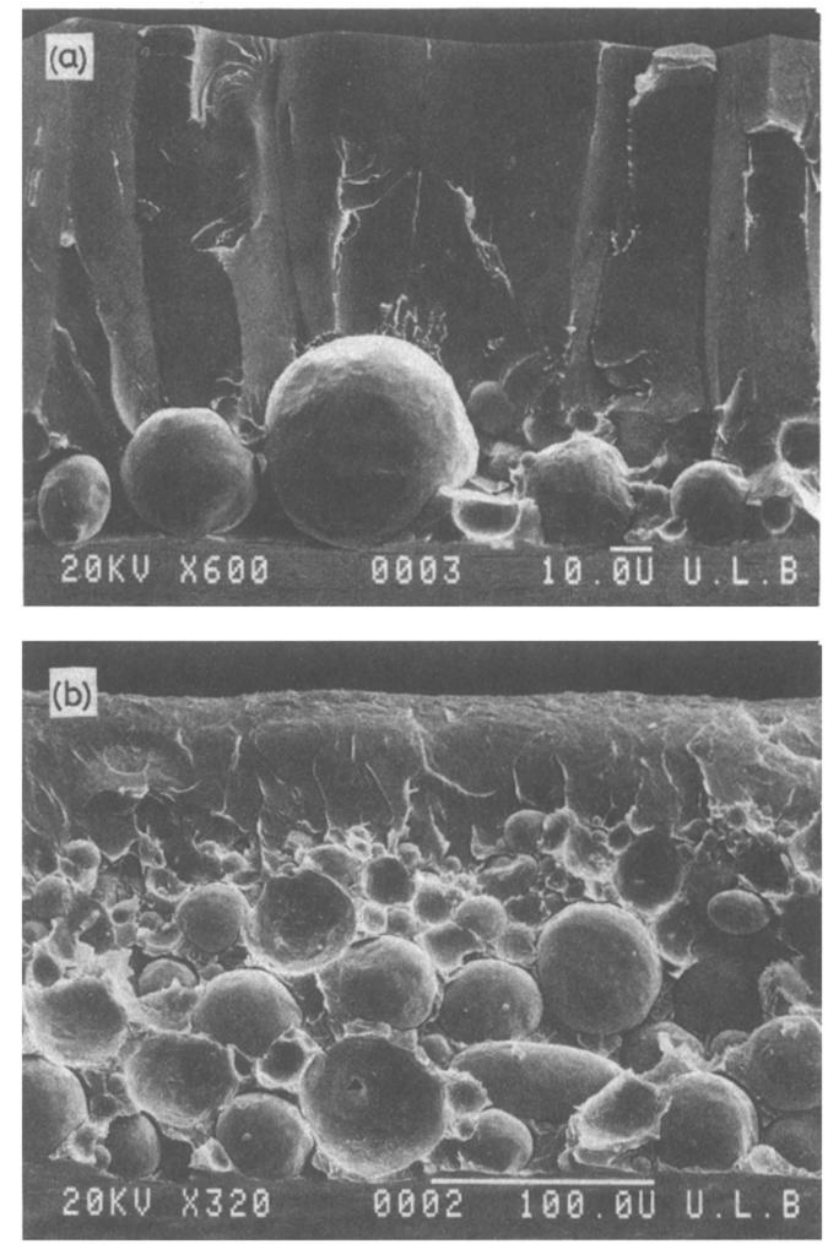

FIG. 2. Cross-sectional views of copper-particle-polymer samples: (a) low-particle-concentration limit (percolationthreshold limit); (b) moderately high particle concentration, just before the particles fill the entire film thickness. 